\title{
Comprehensive Evaluation Method of Transformer Area State Based on Hopfield Neural Network
}

\author{
Dong Wang, Fei Liu and Jing Fu \\ Nantong Power Supply Company, State Grid, Nantong, 226000,China
}

Keywords: Transformer Area Management; Comprehensive Evaluation; Hopfield Neural Network

\begin{abstract}
The intelligent distribution network is a kind of complex system, which is affected by many factors. The establishment of evaluation indexes and method of transformer area is the basis for further scientific planning, construction and management of distribution network. Based on the advantages of neural network in state evaluation, this paper presents a comprehensive evaluation method based on discrete Hopfield neural network. According to the evaluation indexes system, a comprehensive evaluation algorithm is designed. Finally, an example is given to demonstrate the effectiveness and universality of the method..
\end{abstract}

\section{Introduction}

As one part of the smart grid, the distribution network is directly oriented to the users, which is the key link to ensure the quality of power supply and improve the operation efficiency of the power grid [1]. China's low-voltage distribution network has huge and complex structure. With the development of social economy, the electricity load is increasing rapidly. At the same time, the line loss and voltage deviation are more and more outstanding. How to reduce the power loss of distribution network and ensure the power quality in the user side have become the most important task of power supply enterprises.

For power supply enterprises, due to the large number of transformer areas in the low-voltage distribution network and the uneven construction situation, the transformer area management need to be more refined with the increase in electricity supply. Therefore, it is necessary to reasonably evaluate the operation state of every transformer area. The operation state obtained through the quantitative calculation results can provide the basis for the decision-making of the enterprise, which is also the new requirements to distribution network and transformer area management required by the development trend in the distribution automation system [2-4].

\section{Background and Problem Statement}

\section{A. Transformer Area State Management}

Due to the different area and different distribution network construction, it is not reasonable to judge the transformer area in the low-voltage distribution network. We should make distinction requirements in the operation state management. With the continuous increase of the power consumption of low-voltage users, the quality of power supply is constantly improving, the power supply capacity of low-voltage distribution network in some areas has been unable to fully meet the needs of users.

For example, most of the rural area and the city grid of developed area is still a big gap. Rural power grid infrastructure, construction, management system and power market requirements are not suited, can not meet the rapid growth of electricity consumption in rural areas. At present, rural low-voltage distribution network intelligent level is relatively low. Such as low smart meter installation rate, weak power distribution monitoring bring some difficulties to the management.

B. Hopfield Neural Network

A Hopfield net is a recurrent neural network having synaptic connection pattern such that there is an underlying Lyapunov function for the activity dynamics. Started in any initial state, the state of the system evolves to a final state that is a (local) minimum of the Lyapunov function ${ }^{[5-7]}$.

The units in Hopfield nets are binary threshold units, i.e. the units only take on two different 
values for their states and the value is unwavering by whether or not the units' input exceeds their threshold. Hopfield nets normally have units that take on values of 1 or -1 , and this reunion will be used all the way through the piece of writing. Every two units $i$ and $j$ of a Hopfield network have a connection that is described by the connectivity weight. In this sense, the Hopfield network can be formally described as a complete undirected graph $G=<V, f\rangle$, where $V$ is a set of McCulloch-Pitts neurons and is a function that links pairs of nodes to a real value, the connectivity weight.

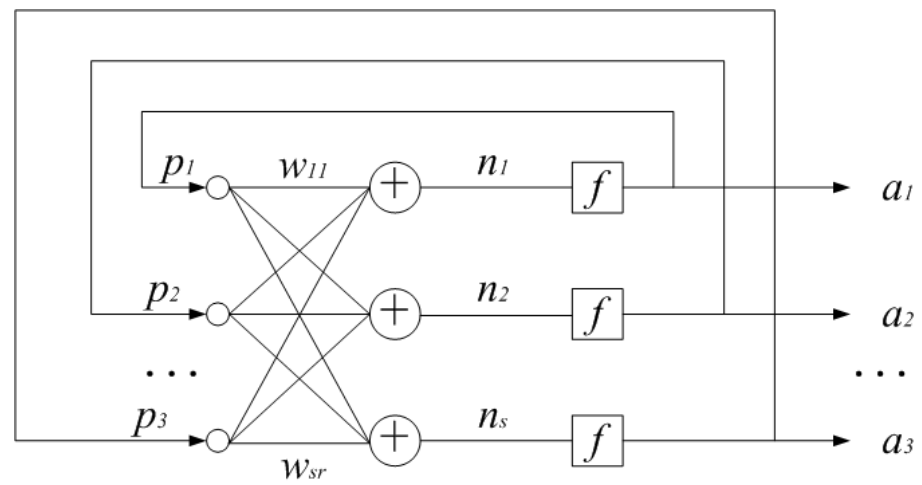

Fig. 1. Architecture of the Hopfield network

There are two popular forms of the model:

(1) Binary neurons with discrete time, updated one at a time.

$V_{j}(t+1)=\left\{\begin{array}{c}1, \text { if } \sum_{k} T_{j k} V_{k}(t)+I_{j}>0 \\ 0, \text { otherwise }\end{array}\right.$

(2) Graded neurons with continuous time.

$d x_{j} / d t=-x_{j} / \tau+\sum_{k} T_{j k} g\left(x_{k}\right)+I_{j}$

Here, $V_{j}$ denotes activity of the $j$-th neuron; $x_{j}$ is the mean internal potential of the neuron; $I_{j}$ is direct input (e.g., sensory input or bias current) to the neuron; $T_{j k}$ is the strength of synaptic input from neuron $k$ to neuron $j ; g$ is a monotone function that converts internal potential into firing rate output of the neuron, i.e., $V_{j}=g\left(x_{j}\right)$.

\section{Comprehensive Evaluation Method Using DHNN}

\section{A. Evaluation Index System}

Considering the integrity and real time of distribution network are till in need of improvement, to facilitate comparison of the applicability and the feasibility of application process, we utilize some basic information to reflect the characteristics of transformer area as literature [2]. Extraction of low-voltage distribution network characteristic index mainly covers characteristics of grid and load. The grid characteristics mainly refers to the line parameters of transformer area, including power supply radius and distribution transformer outlet section; and the load characteristics of transformer area mean the consumption level, including peak average load rate, load rate and growth rate of the annual electricity consumption.

(1) Power supply radius $\left(X_{1}\right)$ : it refers to the most long distance of the power distribution transformer, which is an important parameter influencing the rationality of the structure of the power network. If the power supply radius is too long, it is easy to cause the terminal voltage problem. In general, the transformer area with longer power supply radius.

(2) Distribution transformer outlet section $\left(X_{2}\right)$ : The outlet total section of the distribution transformer is the total section area of the outlet of the distribution transformer in the station area, which reflects the overall condition of the line of the station area.

(3) Peak average load rate(X3): The peak period average load ratio is the distribution 
transformer load ratio in the peak period, which reflects the load weight of the whole transformer area. Usually, the power consumption of the transformer area has obvious seasonal change characteristics, and the user's low voltage problems are mainly concentrated in the peak load period. Therefore, the consideration of the average load rate of the peak period is reasonable.

(4) Load rate $\left(X_{4}\right)$ : It is used to measure the load fluctuation of the district during the whole year. Usually, the higher load rate shows that the annual load is relatively flat. while the lower load rate shows that the load fluctuation is obvious and the operating condition is bad.

(5) Annual electricity consumption growth $\operatorname{rate}\left(X_{5}\right)$ : It is the growth rate of this year's electricity consumption with respect to the last year's electricity consumption, which reflecting the level of load growth in transformer area.

B. Evaluation Algorithm using DHNN

The evaluation index system corresponding to several typical classification level was designed as equilibrium point of discrete Hopfield neural network. The learning process is the typical evaluation index gradually approaching to Hopfield neural network equilibrium.

After the completion of the study process, the equilibrium point of the Hopfield neural network is the evaluation index of each classification level. When the evaluation index is to be classified, the Hopfield neural network is to use the ability of associative memory to gradually close to the stored equilibrium point. When the state is no longer changed, according to the corresponding equilibrium point, we can obtain the final degree of classification.

Generally speaking, the transformer area state comprehensive evaluation algorithm based on Hopfield neural network is shown in the following.

Step 1: Designing the ideal evaluation index system. A feasible method is to use the average value of each evaluation index as an ideal evaluation index of each level, that is, the equilibrium point of the Hopfield neural network.

Step 2: Coding the ideal level evaluation index. Because there are only two state of the discrete Hopfield neural network: 1 and -1 , the evaluation index needs to be coded when it is mapped to the state of the neuron. The coding rule is: when the index is greater than or equal to a certain level, the corresponding neuron's state is set to 1 , otherwise, it is set to -1 .

Step 3: Coding the level evaluation index that waiting for classifying. Similarly, we can get the corresponding coding results according to the above coding rule.

Step 4: Creating a Hopfield neural network. After designing the ideal evaluation index and coding, we can use the neural network toolbox of MATLAB to create the discrete Hopfield neural network.

Step 5: Simulating and analyzing. After the Hopfield neural network has been created, the coding result of the level evaluation index that waiting for classifying was taken as the input data of the neural network. After a certain number of learning process, the simulation results can be obtained.

\section{Performance Evaluation}

\section{A. Ideal Evaluation Index Design}

In order to introduce the evaluation algorithm using DHNN, let us suppose that there are 20 known transformer areas in the distribution network. The 5 evaluation indexes and the evaluation level as shown in the following table 1. 
Table 1: The pretreatment results of evaluation index

\begin{tabular}{ccccccc}
\hline No. & $X_{1}$ & $X_{2}$ & $X_{3}$ & $X_{4}$ & $X_{5}$ & Level \\
\hline 1 & 0.98 & 0.93 & 0.88 & 0.95 & 0.92 & $\mathrm{I}$ \\
2 & 0.94 & 0.96 & 0.94 & 0.90 & 0.95 & $\mathrm{I}$ \\
3 & 0.74 & 0.86 & 0.82 & 0.68 & 0.89 & II \\
4 & 0.78 & 0.72 & 0.76 & 0.90 & 0.82 & II \\
5 & 0.88 & 0.96 & 0.93 & 0.98 & 0.92 & $\mathrm{I}$ \\
6 & 0.68 & 0.72 & 0.66 & 0.64 & 0.69 & $\mathrm{III}$ \\
7 & 0.62 & 0.64 & 0.62 & 0.58 & 0.67 & $\mathrm{III}$ \\
8 & 0.48 & 0.44 & 0.50 & 0.62 & 0.48 & $\mathrm{IV}$ \\
9 & 0.52 & 0.46 & 0.48 & 0.58 & 0.55 & $\mathrm{IV}$ \\
10 & 0.94 & 0.97 & 0.91 & 0.96 & 0.88 & $\mathrm{I}$ \\
11 & 0.26 & 0.36 & 0.44 & 0.32 & 0.18 & $\mathrm{~V}$ \\
12 & 0.84 & 0.82 & 0.72 & 0.78 & 0.73 & $\mathrm{II}$ \\
13 & 0.44 & 0.58 & 0.55 & 0.45 & 0.60 & $\mathrm{IV}$ \\
14 & 0.34 & 0.22 & 0.16 & 0.27 & 0.36 & $\mathrm{~V}$ \\
15 & 0.18 & 0.42 & 0.32 & 0.38 & 0.26 & $\mathrm{~V}$ \\
16 & 0.65 & 0.66 & 0.68 & 0.62 & 0.62 & $\mathrm{III}$ \\
17 & 0.58 & 0.66 & 0.62 & 0.68 & 0.70 & $\mathrm{III}$ \\
18 & 0.74 & 0.84 & 0.94 & 0.78 & 0.84 & $\mathrm{II}$ \\
19 & 0.32 & 0.28 & 0.34 & 0.20 & 0.24 & $\mathrm{~V}$ \\
20 & 0.96 & 0.89 & 0.96 & 0.96 & 0.92 & $\mathrm{I}$ \\
\hline
\end{tabular}

The average value of each evaluation index corresponding to each grade of the sample is the ideal evaluation index of each grade, which is the equilibrium point of the neural network. The results are shown in table 2 .

Table 2: The ideal evaluation index

\begin{tabular}{cccccc}
\hline Level & $X_{1}$ & $X_{2}$ & $X_{3}$ & $X_{4}$ & $X_{5}$ \\
\hline I & 0.94 & 0.94 & 0.92 & 0.95 & 0.92 \\
II & 0.78 & 0.81 & 0.81 & 0.79 & 0.82 \\
III & 0.63 & 0.67 & 0.65 & 0.63 & 0.67 \\
IV & 0.48 & 0.49 & 0.51 & 0.55 & 0.54 \\
V & 0.28 & 0.32 & 0.32 & 0.29 & 0.26 \\
\hline
\end{tabular}

According to the coding rule described in the above evaluation algorithm using DHNN, the coding results of ideal evaluation index as shown in Fig. 2.

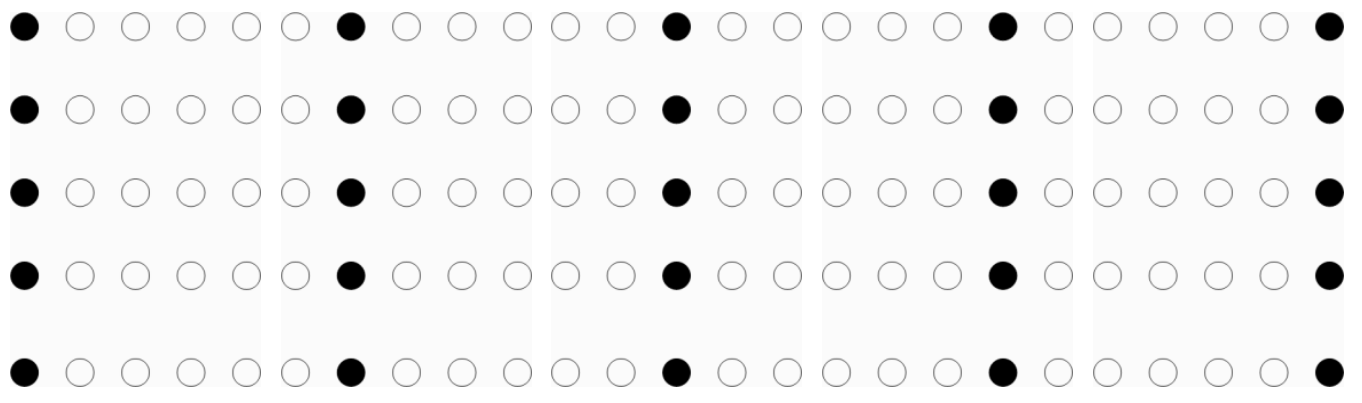

Fig. 2. The coding of ideal evaluation index 


\section{B. DHNN Simulation and Analysis}

Assuming there are 4 transformer area waiting for evaluation in the distribution network. The index data was shown in table 3 . In the same way, according to the above coding algorithm, the results were shown in figure 3.

Table 3: The pretreatment results of evaluation index

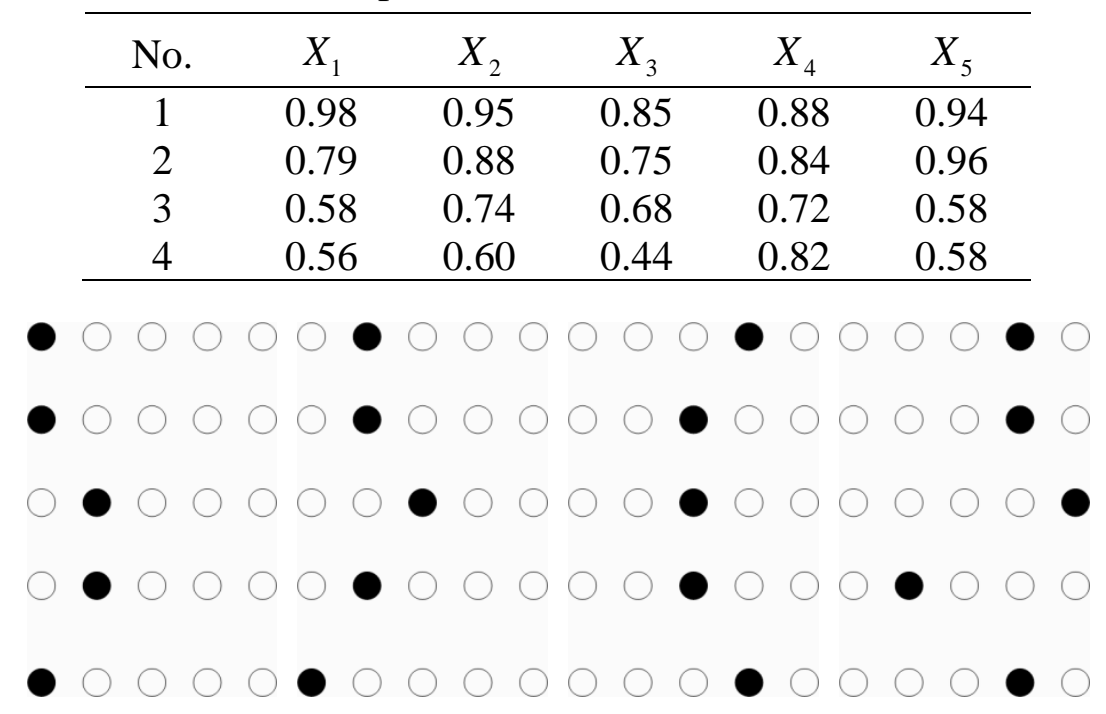

Fig. 3. The coding of evaluation index that waiting for classifying

Figure 4 shows the Hopfield neural network classification results of these four transformer areas. From the figure we can see that the network can effectively classify, so as to make an objective and fair assessment of the state of distribution network in transformer area.

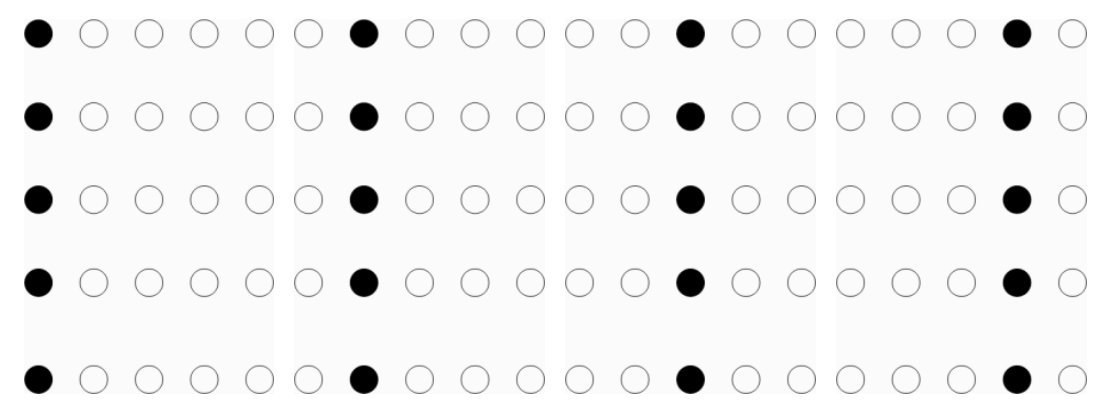

Fig. 4. The classification results by DHNN

\section{Conclusion}

In the face of the small distribution network monitoring configuration, the lack of basic data, the complex state of transformer area line, how to make efficient data collection for a large number of transformer area and quickly determine its state is an urgent problem to be solved. In this paper, we propose comprehensive evaluation method of transformer area state based on Hopfield neural network.

It is worth noting that the discrete Hopfield neural network is not applicable to any occasion. When the quite obvious advantages and disadvantages of the evaluation object coexist, the Hopfield neural network may not be able to classify exactly.

\section{References}

[1] Ni Jingmin, He Guangyu, Shen Chen, et al. A review of assessment of smart grid in America [J], Automation of Electric Power Systems, 2010, 34(8): 9-13 (in Chinese). 
[2] OuYang Sen, Yang Jihao, Geng Hongjie, et al. Comprehensive evaluation method of transformer area state oriented to transformer area management and its application [J], Automation of Electric Power Systems, 2015, 39(11): 187-192 (in Chinese).

[3] Wang Zhidong, Li Hui, Li Jun, et al. Assessment index system for smart grids [J], Power System Technology, 2009, 33(17): 14-18 (in Chinese).

[4] Zhang Xinjie, Ge Shaoyun, Liu Hong, et al. Comprehensive assessment system and method of smart distribution grid [J], Power System Technology, 2014, 38(1): 40-46(in Chinese).

[5] Hopfield J J. Hopfield, J.J.: Neural Network and Physical Systems with Emergent Collective Computational Abilities. Proc. Natl. Acad. Sci. 79, 2554-2558 [J]. Proceedings of the National Academy of Sciences, 1982, 79(8): 2554-8.

[6] Mceliece R J, Posner E C, Rodemich E R, et al. The capacity of the Hopfield associative memory [J]. IEEE Transactions on Information Theory, 1987, 33(4): 461-482.

[7] Fausett L V. Hopfield Neural Networks [M], Wiley Encyclopedia of Computer Science and Engineering. 2008: 21. 